\title{
Certain transformations and summations for generalized hypergeometric series with integral parameter differences
}

\author{
A. R. Miller $\dagger$ \\ Formerly Professor of Mathematics at George Washington University, \\ 1616 18th Street NW, No. 210, Washington, DC 20009-2525, USA \\ and \\ R. B. PARIS \\ Division of Complex Systems, \\ University of Abertay Dundee, Dundee DD1 $1 H G, U K$ \\ r.paris@abertay.ac.uk
}

\begin{abstract}
Certain transformation and summation formulas for generalized hypergeometric series with integral parameter differences are derived.
\end{abstract}

Mathematics Subject Classification: 33C15, 33C20

Keywords: Generalized hypergeometric series, Quadratic transformation, Summation theorems

\section{Introduction}

The generalized hypergeometric function ${ }_{p} F_{q}(x)$ is defined for complex parameters and argument by the series

$$
{ }_{p} F_{q}\left(\begin{array}{c}
a_{1}, a_{2}, \ldots, a_{p} \\
b_{1}, b_{2}, \ldots, b_{q}
\end{array} \mid x\right)=\sum_{k=0}^{\infty} \frac{\left(a_{1}\right)_{k}\left(a_{2}\right)_{k} \ldots\left(a_{p}\right)_{k}}{\left(b_{1}\right)_{k}\left(b_{2}\right)_{k} \ldots\left(b_{q}\right)_{k}} \frac{x^{k}}{k !}
$$

When $q=p$ this series converges for $|x|<\infty$, but when $q=p-1$ convergence occurs when $|x|<1$. However, when only one of the numeratorial parameters $a_{j}$ is a negative integer or zero, then the series always converges since it is simply a polynomial in $x$ of degree $-a_{j}$. In (1.1) the Pochhammer symbol, or ascending factorial, $(a)_{k}$ is defined by $(a)_{0}=1$ and for $k \geq 1$ by $(a)_{k}=a(a+1) \ldots(a+k-1)$. However, for all integers $k$ we write simply

$$
(a)_{k}=\frac{\Gamma(a+k)}{\Gamma(a)} .
$$

In what follows we shall adopt the convention of writing the finite (except where noted otherwise) sequence of parameters $\left(a_{1}, \ldots, a_{p}\right)$ simply by $\left(a_{p}\right)$ and the product of $p$ Pochhammer symbols by

$$
\left(\left(a_{p}\right)\right)_{k} \equiv\left(a_{1}\right)_{k} \ldots\left(a_{p}\right)_{k},
$$

with an empty product $p=0$ reducing to unity.

In [1-4] we derived in various ways transformation formulas for the generalized hypergeometric functions ${ }_{r+1} F_{r+1}(x)$ and ${ }_{r+2} F_{r+1}(x)$, where here and below at least $r$ pairs of numeratorial and 
denominatorial parameters differ by arbitrary positive integers. In particular, in [4] we stated without proof that the generalized hypergeometric function ${ }_{r+1} F_{r+1}(x)$ in which $r+1$ pairs of numeratorial and denominatorial parameters differ by arbitrary positive integers may be written as a product of $e^{x}$ and a certain polynomial in $x$. In Section 3 we shall provide a proof of this result and discuss its implications.

In $[1,4,5]$ we showed that essentially the same methods used to obtain the transformation formulas alluded to above may be employed to deduce the Karlsson-Minton and other more general summation formulas for the ${ }_{r+2} F_{r+1}(1)$ generalized hypergeometric series with unit argument. In the present investigation we shall derive in Section 4 in a similar manner analogous summation formulas for generalized hypergeometric series of the type ${ }_{r+2} F_{r+1}\left(\frac{1}{2}\right)$ with half unit argument. Finally, in Section 5 we shall consider a certain quadratic transformation for the ${ }_{3} F_{2}(x)$ hypergeometric function.

\section{Preliminary results}

We record two lemmas and a theorem that we shall utilize in the sequel. Lemmas 1 and 2 are proved respectively in [1] and [4] and Theorem 1 is proved in [4]. The notation $\left\{\begin{array}{l}n \\ k\end{array}\right\}$ will be employed to denote the Stirling numbers of the second kind. These nonnegative integers represent the number of ways to partition $n$ objects into $k$ nonempty sets and arise for nonnegative integers $n$ in the generating relation $[6$, p. 262]

$$
x^{n}=\sum_{k=0}^{n}\left\{\begin{array}{l}
n \\
k
\end{array}\right\}(-1)^{k}(-x)_{k}, \quad\left\{\begin{array}{l}
n \\
0
\end{array}\right\}=\delta_{0 n},
$$

where $\delta_{0 n}$ is the Kronecker symbol.

Lemma 1. For nonnegative integers $j$ define

$$
S_{j} \equiv \sum_{n=0}^{\infty} n^{j} \frac{\lambda_{n}}{n !}, \quad S_{0} \equiv \sum_{n=0}^{\infty} \frac{\lambda_{n}}{n !},
$$

where the infinite sequence $\left(\lambda_{n}\right)$ is such that $S_{j}$ converges for all $j$. Then

$$
S_{j}=\sum_{k=0}^{j}\left\{\begin{array}{l}
j \\
k
\end{array}\right\} \sum_{n=0}^{\infty} \frac{\lambda_{n+k}}{n !} .
$$

Lemma 2. For nonnegative integer s let $\left(a_{s}\right)$ denote a parameter sequence containing s elements, where when $s=0$ the sequence is empty. Let $\left(a_{s}+k\right)$ denote the sequence when $k$ is added to each element of $\left(a_{s}\right)$. Let $\mathcal{F}(x)$ denote the generalized hypergeometric function with $r$ numeratorial and denominatorial parameters differing by the positive integers $\left(m_{r}\right)$, namely

$$
\mathcal{F}(x) \equiv{ }_{r+s} F_{r+1}\left(\begin{array}{cc}
\left(a_{s}\right), & \left(f_{r}+m_{r}\right) \\
c, & \left(f_{r}\right)
\end{array} \mid x\right),
$$

where by (1.1) convergence of the series representation for the latter occurs in an appropriate domain depending on the values of $s$ and the elements of the parameter sequence $\left(a_{s}\right)$. Then

$$
\mathcal{F}(x)=\frac{1}{A_{0}} \sum_{k=0}^{m} x^{k} A_{k} \frac{\left(\left(a_{s}\right)\right)_{k}}{(c)_{k}}{ }_{s} F_{1}\left(\begin{array}{c}
\left(a_{s}+k\right) \\
c+k
\end{array} \mid x\right),
$$

where $m=m_{1}+\cdots+m_{r}$, the coefficients $A_{k}$ are defined by

$$
A_{k} \equiv \sum_{j=k}^{m}\left\{\begin{array}{l}
j \\
k
\end{array}\right\} \sigma_{m-j}, \quad A_{0}=\left(f_{1}\right)_{m_{1}} \ldots\left(f_{r}\right)_{m_{r}}, \quad A_{m}=1
$$


and the $\sigma_{j}(0 \leq j \leq m)$ are generated by the relation

$$
\left(f_{1}+x\right)_{m_{1}} \cdots\left(f_{r}+x\right)_{m_{r}}=\sum_{j=0}^{m} \sigma_{m-j} x^{j} .
$$

Theorem 1. Let $\left(m_{r}\right)$ be a nonempty sequence of positive integers and define $m \equiv m_{1}+\cdots+m_{r}$. Then if $b \neq f_{j}(1 \leq j \leq r),(\lambda)_{m} \neq 0$, where $\lambda \equiv c-b-m$, we have the transformation formula

$$
{ }_{r+1} F_{r+1}\left(\begin{array}{cc}
b, & \left(f_{r}+m_{r}\right) \\
c, & \left(f_{r}\right)
\end{array} \mid x\right)=e^{x}{ }_{m+1} F_{m+1}\left(\begin{array}{cc}
\lambda, & \left(\xi_{m}+1\right) \\
c, & \left(\xi_{m}\right)
\end{array} \mid-x\right),
$$

where $|x|<\infty$. The $\left(\xi_{m}\right)$ are the nonvanishing zeros of the associated parametric polynomial $Q_{m}(t)$ of degree $m$ given by

$$
Q_{m}(t)=\sum_{j=0}^{m} \sigma_{m-j} \sum_{k=0}^{j}\left\{\begin{array}{l}
j \\
k
\end{array}\right\}(b)_{k}(t)_{k}(\lambda-t)_{m-k},
$$

where the $\sigma_{j}(0 \leq j \leq m)$ are determined by the generating relation (2.2).

In the following Section 3 we shall consider the generalized hypergeometric function $w(x)$ defined for $|x|<\infty$ by

$$
w(x) \equiv{ }_{r+1} F_{r+1}\left(\begin{array}{c}
\left(f_{r+1}+m_{r+1}\right) \\
\left(f_{r+1}\right)
\end{array} \mid x\right),
$$

where $\left(m_{r+1}\right)$ is a sequence of positive integers. It is evident that $w(x)$ is an entire function.

\section{Properties of $w(x)$}

If in Theorem 1 we set $b=f_{r+1}+m_{r+1}, c=f_{r+1}$ and define $m \equiv m_{1}+\cdots+m_{r}, M \equiv m+m_{r+1}$, then $\lambda=-M,(-M)_{m} \neq 0$ and we find from (2.3) that

$$
w(x)=e^{x}{ }_{m+1} F_{m+1}\left(\begin{array}{cc}
-M, & \left(\xi_{m}+1\right) \\
f_{r+1}, & \left(\xi_{m}\right)
\end{array} \mid-x\right) .
$$

The $\left(\xi_{m}\right)$ are the nonvanishing zeros of the associated parametric polynomial of degree $m$ given by

$$
Q_{m}(t)=\sum_{j=0}^{m} \sigma_{m-j} \sum_{k=0}^{j}\left\{\begin{array}{l}
j \\
k
\end{array}\right\}\left(f_{r+1}+m_{r+1}\right)_{k}(t)_{k}(-M-t)_{m-k},
$$

where the $\sigma_{j}(0 \leq j \leq m)$ are generated by $(2.2)$.

Thus it is evident that $w(x)$ is proportional to a polynomial in $x$ of degree at most $M$ which we define as

$$
\mathcal{P}_{M}(x) \equiv{ }_{m+1} F_{m+1}\left(\begin{array}{cc}
-M, & \left(\xi_{m}+1\right) \\
f_{r+1}, & \left(\xi_{m}\right)
\end{array} \mid-x\right) .
$$

Moreover, since $e^{x}$ can never vanish it follows that the entire function $w(x)$ has at most $M$ zeros in the complex plane. However, we shall obtain an explicit representation for $\mathcal{P}_{M}(x)$ which shows that its degree is exactly $M$.

Theorem 2. Let $\left(m_{r+1}\right)$ be a sequence of positive integers such that $M \equiv m_{1}+\cdots+m_{r+1}$ and let $\left(f_{r+1}\right)$ be a sequence of complex numbers such that $\left(f_{1}\right)_{m_{1}} \ldots\left(f_{r+1}\right)_{m_{r+1}}$ is nonvanishing. Then

$$
w(x)=e^{x} \mathcal{P}_{M}(x),
$$


where $\mathcal{P}_{M}(x)$ is a polynomial of degree $M$ given by

$$
\mathcal{P}_{M}(x)=\frac{1}{B_{0}} \sum_{k=0}^{M} B_{k} x^{k}, \quad B_{k} \equiv \sum_{j=k}^{M}\left\{\begin{array}{l}
j \\
k
\end{array}\right\} \rho_{M-j} .
$$

Here

$$
B_{0}=\left(f_{1}\right)_{m_{1}} \ldots\left(f_{r+1}\right)_{m_{r+1}}, \quad B_{M}=1,
$$

and the $\rho_{j}(0 \leq j \leq M)$ are generated by the relation

$$
\left(f_{1}+x\right)_{m_{1}} \ldots\left(f_{r+1}+x\right)_{m_{r+1}}=\sum_{j=0}^{M} \rho_{M-j} x^{j} .
$$

Proof: Note that

$$
\frac{\left(\left(f_{r+1}+m_{r+1}\right)\right)_{n}}{\left(\left(f_{r+1}\right)\right)_{n}}=\frac{\left(f_{1}+n\right)_{m_{1}}}{\left(f_{1}\right)_{m_{1}}} \ldots \frac{\left(f_{r+1}+n\right)_{m_{r+1}}}{\left(f_{r}\right)_{m_{r+1}}}
$$

where the numeratorial expression on the right-hand side of the latter may be written as

$$
\left(f_{1}+n\right)_{m_{1}} \ldots\left(f_{r+1}+n\right)_{m_{r+1}}=\sum_{j=0}^{M} \rho_{M-j} n^{j} .
$$

Thus by (1.1)

$$
\begin{gathered}
w(x) \equiv{ }_{r+1} F_{r+1}\left(\begin{array}{c}
\left(f_{r+1}+m_{r+1}\right) \\
\left(f_{r+1}\right)
\end{array} \mid x\right)=\sum_{n=0}^{\infty} \frac{\left(\left(f_{r+1}+m_{r+1}\right)\right)_{n}}{\left(\left(f_{r+1}\right)\right)_{n}} \frac{x^{n}}{n !} \\
=\frac{1}{\left(f_{1}\right)_{m_{1}} \ldots\left(f_{r+1}\right)_{m_{r+1}}} \sum_{j=0}^{M} \rho_{M-j} \sum_{n=0}^{\infty} n^{j} \frac{x^{n}}{n !}
\end{gathered}
$$

where the order of the summations has been interchanged.

Now employing Lemma 1 we have

$$
\sum_{n=0}^{\infty} n^{j} \frac{x^{n}}{n !}=\sum_{k=0}^{j}\left\{\begin{array}{l}
j \\
k
\end{array}\right\} \sum_{n=0}^{\infty} \frac{x^{n+k}}{n !}=e^{x} \sum_{k=0}^{j}\left\{\begin{array}{l}
j \\
k
\end{array}\right\} x^{k}
$$

so that

$$
w(x)=\frac{e^{x}}{\left(f_{1}\right)_{m_{1}} \ldots\left(f_{r+1}\right)_{m_{r+1}}} \sum_{j=0}^{M} \rho_{M-j} \sum_{k=0}^{j}\left\{\begin{array}{l}
j \\
k
\end{array}\right\} x^{k},
$$

where

$$
\sum_{j=0}^{M} \rho_{M-j} \sum_{k=0}^{j}\left\{\begin{array}{l}
j \\
k
\end{array}\right\} x^{k}=\sum_{k=0}^{M}\left(\sum_{j=k}^{M}\left\{\begin{array}{l}
j \\
k
\end{array}\right\} \rho_{M-j}\right) x^{k} .
$$

Defining

$$
B_{k} \equiv \sum_{j=k}^{M}\left\{\begin{array}{l}
j \\
k
\end{array}\right\} \rho_{M-j} \quad(0 \leq k \leq M),
$$

we note that when $k=0$ the only contribution to the $j$-summation comes from $j=0$, so that by using (3.1) we find

$$
B_{0}=\rho_{M}=\left(f_{1}\right)_{m_{1}} \ldots\left(f_{r+1}\right)_{m_{r+1}} .
$$


In addition when $k=M$, the only contribution to the latter summation comes from $j=M$, so that by again using (3.1) we see that

$$
B_{M}=\rho_{0}=1
$$

Thus

$$
w(x)=\frac{e^{x}}{B_{0}} \sum_{k=0}^{M} B_{k} x^{k}
$$

which evidently completes the proof.

Thus we also have the following.

Corollary 1. The entire function $w(x)$ has exactly $M$ zeros in the complex plane.

We remark that Ki and Kim [7] only show the existence of at most $M$ zeros for $w(x)$, whereas from (3.2) we can in principle obtain all of the $M$ zeros. For example,

$$
{ }_{2} F_{2}\left(\begin{array}{c|c}
f+1, g+1 \\
f, & g
\end{array}\right)=e^{x}\left(\frac{1}{f g} x^{2}+\frac{f+g+1}{f g} x+1\right),
$$

and here the zeros of $\mathcal{P}_{2}(x)$ are

$$
x_{1,2}=-\frac{1}{2}\left(f+g+1 \pm\left[(f-g)^{2}+2 f+2 g+1\right]^{1 / 2}\right) .
$$

\section{Summation formulas for ${ }_{r+2} F_{r+1}\left(\frac{1}{2}\right)$}

In [4] we employed Lemma 2 to readily obtain a generalization of the Karlsson-Minton summation formula which is given in the following.

Theorem 3. Suppose $\left(m_{r}\right)$ is a sequence of positive integers such that $m=m_{1}+\cdots+m_{r}$. Then provided that $\operatorname{Re}(c-a-b)>m$ we have

$$
{ }_{r+2} F_{r+1}\left(\begin{array}{cc}
a, b, & \left(f_{r}+m_{r}\right) \\
c, & \left(f_{r}\right)
\end{array} \mid 1\right)=\frac{\Gamma(c) \Gamma(c-a-b)}{\Gamma(c-a) \Gamma(c-b)} \sum_{k=0}^{m} \frac{A_{k}}{A_{0}} \frac{(-1)^{k}(a)_{k}(b)_{k}}{(1+a+b-c)_{k}}
$$

where the $A_{k}(0 \leq k \leq m)$ are given by (2.1).

However we may also utilize Lemma 2 to obtain summation formulas for ${ }_{r+2} F_{r+1}\left(\frac{1}{2}\right)$. To this end we note the following (see, for example, $\left[8\right.$, Section 7.3.7 (3)-(6)]) summations for ${ }_{2} F_{1}\left(\frac{1}{2}\right)$ given by

$$
\begin{aligned}
{ }_{2} F_{1}\left(\begin{array}{c|c}
a, b \\
\frac{1}{2} a+\frac{1}{2} b-\frac{1}{2}
\end{array} \mid \frac{1}{2}\right) & =\sqrt{\pi}\left\{\frac{\Gamma\left(\frac{1}{2} a+\frac{1}{2} b+\frac{1}{2}\right)}{\Gamma\left(\frac{1}{2} a+\frac{1}{2}\right) \Gamma\left(\frac{1}{2} b+\frac{1}{2}\right)}+\frac{2 \Gamma\left(\frac{1}{2} a+\frac{1}{2} b-\frac{1}{2}\right)}{\Gamma(a) \Gamma(b)}\right\}, \\
{ }_{2} F_{1}\left(\begin{array}{c}
a, b \\
\frac{1}{2} a+\frac{1}{2} b
\end{array} \mid \frac{1}{2}\right) & =\sqrt{\pi}\left\{\frac{\Gamma\left(\frac{1}{2} a+\frac{1}{2} b\right)}{\Gamma\left(\frac{1}{2} b\right) \Gamma\left(\frac{1}{2} a+\frac{1}{2}\right)}+\frac{\Gamma\left(\frac{1}{2} a+\frac{1}{2} b\right)}{\Gamma\left(\frac{1}{2} a\right) \Gamma\left(\frac{1}{2} b+\frac{1}{2}\right)}\right\}, \\
{ }_{2} F_{1}\left(\begin{array}{c}
a, b \\
\frac{1}{2} a+\frac{1}{2} b+\frac{1}{2}
\end{array} \mid \frac{1}{2}\right) & =\sqrt{\pi} \frac{\Gamma\left(\frac{1}{2} a+\frac{1}{2} b+\frac{1}{2}\right)}{\Gamma\left(\frac{1}{2} a+\frac{1}{2}\right) \Gamma\left(\frac{1}{2} b+\frac{1}{2}\right)}, \\
{ }_{2} F_{1}\left(\begin{array}{c}
a, b \\
\frac{1}{2} a+\frac{1}{2} b+1
\end{array} \mid \frac{1}{2}\right) & =\frac{2 \sqrt{\pi}}{a-b}\left\{\frac{\Gamma\left(\frac{1}{2} a+\frac{1}{2} b+1\right)}{\Gamma\left(\frac{1}{2} a\right) \Gamma\left(\frac{1}{2} b+\frac{1}{2}\right)}-\frac{\Gamma\left(\frac{1}{2} a+\frac{1}{2} b+1\right)}{\Gamma\left(\frac{1}{2} b\right) \Gamma\left(\frac{1}{2} a+\frac{1}{2}\right)}\right\} .
\end{aligned}
$$


Setting $x=\frac{1}{2}, s=2,\left(a_{s}\right)=(a, b), c=\frac{1}{2}(a+b+n)$, where $n=-1,0,1,2$, in the first two equations of Lemma 2, we see that

$$
\begin{array}{r}
{ }_{r+2} F_{r+1}\left(\begin{array}{cc|c}
a, b, & \left(f_{r}+m_{r}\right) & \frac{1}{2} \\
\frac{1}{2} a+\frac{1}{2} b+\frac{1}{2} n, & \left(f_{r}\right)
\end{array}\right) \\
=\sum_{k=0}^{m}\left(\frac{1}{2}\right)^{k} \frac{A_{k}}{A_{0}} \frac{(a)_{k}(b)_{k}}{\left(\frac{1}{2} a+\frac{1}{2} b+\frac{1}{2} n\right)_{k}}{ }_{2} F_{1}\left(\begin{array}{c}
a+k, b+k \\
\frac{1}{2} a+\frac{1}{2} b+\frac{1}{2} n+k
\end{array} \mid \frac{1}{2}\right),
\end{array}
$$

where when $r=0,\left(f_{r}\right)$ and $\left(f_{r}+m_{r}\right)$ are empty and we define $m \equiv 0$. Thus the Gauss series ${ }_{2} F_{1}\left(\frac{1}{2}\right)$ in (4.5) with respectively $n=-1,0,1,2$ correspond to the left-hand sides of (4.1)-(4.4) with $a \mapsto a+k$ and $b \mapsto b+k$. Combining these with (4.5) we therefore deduce the following.

Theorem 4. Let $\left(m_{r}\right)$ be an arbitrary sequence of positive integers such that $m \equiv m_{1}+\cdots+m_{r}$ and $\left(f_{r}\right)$ a sequence of complex numbers such that $\left(f_{1}\right)_{m_{1}} \ldots\left(f_{r}\right)_{m_{r}} \neq 0$. Then

$$
\begin{aligned}
& { }_{r+2} F_{r+1}\left(\begin{array}{cc|c}
a, b, & \left(f_{r}+m_{r}\right) & \frac{1}{2} \\
\frac{1}{2} a+\frac{1}{2} b-\frac{1}{2}, & \left(f_{r}\right)
\end{array}\right. \\
& =\frac{2 \sqrt{\pi}}{A_{0}} \frac{\Gamma\left(\frac{1}{2} a+\frac{1}{2} b-\frac{1}{2}\right)}{\Gamma(a) \Gamma(b)} \sum_{k=0}^{m}\left(\frac{1}{2}\right)^{k} A_{k}\left\{1+\frac{\Gamma(a+k) \Gamma(b+k)(a+b-1+2 k)}{4 \Gamma\left(\frac{1}{2} a+\frac{1}{2}+\frac{1}{2} k\right) \Gamma\left(\frac{1}{2} b+\frac{1}{2}+\frac{1}{2} k\right)}\right\}, \\
& { }_{r+2} F_{r+1}\left(\begin{array}{cc|c}
a, b, & \left(f_{r}+m_{r}\right) & \frac{1}{2} \\
\frac{1}{2} a+\frac{1}{2} b, & \left(f_{r}\right)
\end{array}\right) \\
& =\frac{2^{a+b-2}}{A_{0} \sqrt{\pi}} \frac{\Gamma\left(\frac{1}{2} a+\frac{1}{2} b\right)}{\Gamma(a) \Gamma(b)} \sum_{k=0}^{m} 2^{k} A_{k}\left\{\Gamma\left(\frac{1}{2} a+\frac{1}{2} k\right) \Gamma\left(\frac{1}{2} b+\frac{1}{2}+\frac{1}{2} k\right)-\Gamma\left(\frac{1}{2} b+\frac{1}{2} k\right) \Gamma\left(\frac{1}{2} a+\frac{1}{2}+\frac{1}{2} k\right)\right\}, \\
& { }_{r+2} F_{r+1}\left(\begin{array}{cc|c}
a, b, & \left(f_{r}+m_{r}\right) & \frac{1}{2} \\
\frac{1}{2} a+\frac{1}{2} b+\frac{1}{2}, & \left(f_{r}\right) &
\end{array}\right. \\
& =\frac{2^{a+b-2}}{A_{0} \sqrt{\pi}} \frac{\Gamma\left(\frac{1}{2} a+\frac{1}{2} b+\frac{1}{2}\right)}{\Gamma(a) \Gamma(b)} \sum_{k=0}^{m} 2^{k} A_{k} \Gamma\left(\frac{1}{2} a+\frac{1}{2} k\right) \Gamma\left(\frac{1}{2} b+\frac{1}{2} k\right), \\
& { }_{r+2} F_{r+1}\left(\begin{array}{cc|c}
a, b, & \left(f_{r}+m_{r}\right) & \frac{1}{2} \\
\frac{1}{2} a+\frac{1}{2} b+1, & \left(f_{r}\right) &
\end{array}\right) \\
& =\frac{2^{a+b-2}}{A_{0} \sqrt{\pi}} \frac{a+b}{a-b} \frac{\Gamma\left(\frac{1}{2} a+\frac{1}{2} b\right)}{\Gamma(a) \Gamma(b)} \sum_{k=0}^{m} 2^{k} A_{k}\left\{\Gamma\left(\frac{1}{2} b+\frac{1}{2} k\right) \Gamma\left(\frac{1}{2} a+\frac{1}{2} k+\frac{1}{2}\right)-\Gamma\left(\frac{1}{2} a+\frac{1}{2} k\right) \Gamma\left(\frac{1}{2} b+\frac{1}{2} k+\frac{1}{2}\right)\right\},
\end{aligned}
$$

where the $A_{k}(0 \leq k \leq m)$ are given by (2.1).

We have used the duplication formula

$$
\sqrt{\pi} \Gamma(2 z)=2^{2 z-1} \Gamma(z) \Gamma\left(z+\frac{1}{2}\right)
$$

to obtain (4.7)-(4.9). Thus, when $r=0$, the sequences $\left(f_{r}\right)$ and $\left(f_{r}+m_{r}\right)$ are empty so that $m=0$ and (4.6)-(4.9) reduce respectively to (4.1)-(4.4). Since (4.9) is not valid when $a=b$ we may use l'Hôpital's rule and (4.10) to obtain the limiting case of (4.9), namely

$$
{ }_{r+2} F_{r+1}\left(\begin{array}{cc}
a, a,\left(f_{r}+m_{r}\right) \\
a+1, & \left(f_{r}\right)
\end{array} \mid \frac{1}{2}\right)=\frac{2^{a-1} a}{A_{0}} \sum_{k=0}^{m} A_{k}(a) k\left\{\psi\left(\frac{1}{2} a+\frac{1}{2} k+\frac{1}{2}\right)-\psi\left(\frac{1}{2} a+\frac{1}{2} k\right)\right\},
$$


where $\psi$ is the digamma or psi function. When $r=0$ we retrieve

$$
{ }_{2} F_{1}\left(\begin{array}{c|c}
a, a \\
a+1
\end{array} \mid \frac{1}{2}\right)=2^{a-1} a\left\{\psi\left(\frac{1}{2} a+\frac{1}{2}\right)-\psi\left(\frac{1}{2} a\right)\right\}
$$

which is found in $[8$, Section $7.3 .7(16)]$ in an equivalent form.

In addition, if we let $r=1, m_{1}=1$ and $f_{1}=f$, so that $A_{0}=f, A_{1}=1$, then we obtain from (4.9) and (4.10) after some straightforward algebra the result (when $a \neq b$ )

$$
{ }_{3} F_{2}\left(\begin{array}{c|c}
a, b, \quad f+1 & \frac{1}{2} \\
\frac{1}{2} a+\frac{1}{2} b+1, & f
\end{array}\right)=\sqrt{\pi}\left(\frac{a+b}{a-b}\right) \Gamma\left(\frac{1}{2} a+\frac{1}{2} b\right)\left\{\frac{(a-f) / f}{\Gamma\left(\frac{1}{2} b\right) \Gamma\left(\frac{1}{2} a+\frac{1}{2}\right)}-\frac{(b-f) / f}{\Gamma\left(\frac{1}{2} a\right) \Gamma\left(\frac{1}{2} b+\frac{1}{2}\right)}\right\}
$$

which has been obtained by Rathie and Pogány in [9].

\section{A quadratic transformation for ${ }_{3} F_{2}(x)$}

In [4] by utilizing Lemma 2 we derived two quadratic transformations for

$$
{ }_{r+2} F_{r+1}\left(\begin{array}{cc}
a, a+\frac{1}{2}, & \left(f_{r}+m_{r}\right) \\
c, & \left(f_{r}\right)
\end{array}\right)
$$

where $X \equiv x^{2} /(1 \pm x)^{2}$ and $X \equiv 4 x /(1+x)^{2}$. As discussed below certain parametric conditions guaranteeing the existence of a quadratic transformation for the Gauss function ${ }_{2} F_{1}(x)$ restrict the existence of more general quadratic transformations for ${ }_{r+2} F_{r+1}(x)$ when the decomposition Lemma 2 is employed. We illustrate this by deducing a quadratic transformation that necessarily is restricted to $r=1$ thus giving the quadratic transformation for ${ }_{3} F_{2}(x)$ in the following theorem.

Theorem 5. Suppose $a, b \neq \frac{1}{2}$ and $f \neq a+b+\frac{1}{2}$. Then

$$
\begin{aligned}
& { }_{3} F_{2}\left(\begin{array}{cc|c}
a, b, & f+1 & 4 x(1-x)) \\
a+b+\frac{1}{2}, & f & 4 x(1-2)
\end{array}\right. \\
& =(1-2 x)^{-1}{ }_{4} F_{3}\left(\begin{array}{ccc}
2 a-1,2 b-1, & \xi_{1}+1, & \xi_{2}+1 \\
a+b+\frac{1}{2}, & \xi_{1}, & \xi_{2}
\end{array} \mid x\right),
\end{aligned}
$$

where $\xi_{1}, \xi_{2}$ are given by

$$
\xi_{1,2}=\alpha+\frac{1}{2} \pm\left[\left(\alpha+\frac{1}{2}\right)^{2}-2 \alpha f\right]^{1 / 2}, \quad \alpha=\frac{(2 a-1)(2 b-1)}{2(a+b-f)-1} .
$$

The transformation (5.1) holds in a neighborhood of $x=0$.

Proof: From Lemma 2 we have the expansion of ${ }_{r+2} F_{r+1}(z)$, with $r$ pairs of parameters differing by the positive integers $\left(m_{r}\right)$, as a finite sum of ${ }_{2} F_{1}(z)$ functions in the form

$$
{ }_{r+2} F_{r+1}\left(\begin{array}{cc}
a, b, & \left(\begin{array}{c}
\left.f_{r}+m_{r}\right) \\
c,
\end{array}\left(f_{r}\right)\right.
\end{array} \mid z\right)=\frac{1}{A_{0}} \sum_{k=0}^{m} z^{k} A_{k} \frac{(a)_{k}(b)_{k}}{(c)_{k}}{ }_{2} F_{1}\left(\begin{array}{c}
a+k, b+k \\
c+k
\end{array} \mid z\right)
$$

where the coefficients $A_{k}$ are defined by (2.1). If we set $r=1, m_{1}=1$ and $f_{1}=f$ in the above expansion we obtain the particular case

$$
{ }_{3} F_{2}\left(\begin{array}{cc|c}
a, b, & f+1 \\
c, & f & z
\end{array}\right)={ }_{2} F_{1}\left(\begin{array}{c}
a, b \\
c
\end{array} \mid z\right)+\frac{a b z}{c f}{ }_{2} F_{1}\left(\begin{array}{c}
a+1, b+1 \\
c+1
\end{array} \mid z\right) .
$$


Now let $z=X \equiv 4 x(1-x)$ and define

$$
F(x) \equiv{ }_{3} F_{2}\left(\begin{array}{cc|c}
a, b, & f+1 & X \\
a+b+\frac{1}{2}, & f &
\end{array}\right)
$$

Then, from (5.4) with $c=a+b+\frac{1}{2}$, we find

$$
F(x)={ }_{2} F_{1}\left(\begin{array}{c|c}
a, b \\
a+b+\frac{1}{2}
\end{array} \mid X\right)+\frac{a b}{c f} X_{2} F_{1}\left(\begin{array}{c}
a+1, b+1 \\
a+b+\frac{3}{2}
\end{array} \mid X\right) .
$$

We now make use of two quadratic transformation formulas for the Gauss hypergeometric function $[10$, Section $15.3,(22)$ and (24)], namely

$$
{ }_{2} F_{1}\left(\begin{array}{c}
a, b \\
a+b-j+\frac{1}{2}
\end{array} \mid X\right)=(1-2 x)^{-j}{ }_{2} F_{1}\left(\begin{array}{c}
2 a-j, 2 b-j \\
a+b-j+\frac{1}{2}
\end{array} \mid x\right),
$$

where $j=0,1$. Application of these transformations with $j=0$ and $j=1$ respectively to the first and second hypergeometric functions in (5.5) then leads to

$$
\begin{aligned}
F(x)=(1-2 x)^{-1}\left\{(1-2 x){ }_{2} F_{1}\left(\begin{array}{c}
2 a, 2 b \\
a+b+\frac{1}{2}
\end{array} \mid x\right)+\right. \\
\left.\frac{4 a b}{c f} x(1-x)_{2} F_{1}\left(\begin{array}{c|c}
2 a+1,2 b+1 \\
a+b+\frac{3}{2}
\end{array}\right)\right\} .
\end{aligned}
$$

From (1.1) it can be seen that for arbitrary values of $c$

$$
\begin{aligned}
& { }_{2} F_{1}\left(\begin{array}{c|c}
2 a, 2 b & x \\
c & x
\end{array}\right)=\sum_{n=0}^{\infty} \frac{(2 a)_{n}(2 b)_{n}}{(c)_{n}} \frac{x^{n}}{n !} \\
& =\sum_{n=0}^{\infty} \frac{(2 a-1)_{n}(2 b-1)_{n}}{(c)_{n}} \frac{x^{n}}{n !} \frac{(2 a-1+n)(2 b-1+n)}{(2 a-1)(2 b-1)}, \\
& x_{2} F_{1}\left(\begin{array}{c|c}
2 a, 2 b & x \\
c & x
\end{array}\right)=\sum_{n=1}^{\infty} \frac{(2 a)_{n-1}(2 b)_{n-1}}{(c)_{n-1}} \frac{x^{n}}{(n-1) !} \\
& =\sum_{n=0}^{\infty} \frac{(2 a-1)_{n}(2 b-1)_{n}}{(c)_{n}} \frac{x^{n}}{n !} \frac{n(c-1+n)}{(2 a-1)(2 b-1)}, \\
& x_{2} F_{1}\left(\begin{array}{c|c}
2 a+1,2 b+1 \\
c+1
\end{array} \mid x\right)=\sum_{n=1}^{\infty} \frac{(2 a+1)_{n-1}(2 b+1)_{n-1}}{(c+1)_{n-1}} \frac{x^{n}}{(n-1) !} \\
& =\frac{c}{4 a b} \sum_{n=0}^{\infty} \frac{(2 a-1)_{n}(2 b-1)_{n}}{(c)_{n}} \frac{x^{n}}{n !} \frac{n(2 a-1+n)(2 b-1+n)}{(2 a-1)(2 b-1)},
\end{aligned}
$$

and

$$
\begin{aligned}
x^{2}{ }_{2} F_{1}\left(\begin{array}{c}
2 a+1,2 b+1 \\
c+1
\end{array} \mid x\right) & =\sum_{n=2}^{\infty} \frac{(2 a+1)_{n-2}(2 b+1)_{n-2}}{(c+1)_{n-2}} \frac{x^{n}}{(n-2) !} \\
& =\frac{c}{4 a b} \sum_{n=0}^{\infty} \frac{(2 a-1)_{n}(2 b-1)_{n}}{(c)_{n}} \frac{x^{n}}{n !} \frac{n(n-1)(c-1+n)}{(2 a-1)(2 b-1)}
\end{aligned}
$$


where obvious adjustments to the summation index $n$ have been made. Then, upon setting $c=a+b+\frac{1}{2}$ in the above sums, we obtain from (5.7)

$$
F(x)=\frac{(1-2 x)^{-1}}{(2 a-1)(2 b-1)} \sum_{n=0}^{\infty} \frac{(2 a-1)_{n}(2 b-1)_{n}}{\left(a+b+\frac{1}{2}\right)_{n}} \frac{x^{n}}{n !} P_{2}(n),
$$

where, with $\gamma \equiv f^{-1}\left(a+b-\frac{1}{2}\right)-1$,

$$
\begin{aligned}
P_{2}(n) & =(2 a-1+n)(2 b-1+n)\left(1+\frac{n}{f}\right)-n\left(2+\frac{n-1}{f}\right)\left(a+b-\frac{1}{2}+n\right) \\
& =\gamma n^{2}+\left(\gamma+\frac{1}{f}(2 a-1)(2 b-1)\right) n+(2 a-1)(2 b-1) .
\end{aligned}
$$

The quadratic $P_{2}(n)$ can be factored to yield

$$
P_{2}(n)=\gamma\left(n+\xi_{1}\right)\left(n+\xi_{2}\right),
$$

where, provided $a, b \neq \frac{1}{2}$ and $f \neq a+b-\frac{1}{2}, \xi_{1}$ and $\xi_{2}$ are given by (5.2). Since $\gamma \xi_{1} \xi_{2}=$ $(2 a-1)(2 b-1)$, we therefore see that

$$
\begin{aligned}
F(x) & =(1-2 x)^{-1} \sum_{n=0}^{\infty} \frac{(2 a-1)_{n}(2 b-1)_{n}}{\left(a+b+\frac{1}{2}\right)_{n}} \frac{x^{n}}{n !} \frac{\left(n+\xi_{1}\right)\left(n+\xi_{2}\right)}{\xi_{1} \xi_{2}} \\
& =(1-2 x)^{-1} \sum_{n=0}^{\infty} \frac{(2 a-1)_{n}(2 b-1)_{n}}{\left(a+b+\frac{1}{2}\right)_{n}} \frac{\left(\xi_{1}+1\right)_{n}\left(\xi_{2}+1\right)_{n}}{\left(\xi_{1}\right)_{n}\left(\xi_{2}\right)_{n}} \frac{x^{n}}{n !},
\end{aligned}
$$

where we have made use of the fact that $(\lambda+1)_{n} /(\lambda)_{n}=(n+\lambda) / \lambda$. This yields (5.1) and so completes the proof.

We remark that when $f=a+b-\frac{1}{2}$, the coefficient $\gamma=0$ and the polynomial $P_{2}(n)$ becomes linear with $\xi_{1}=f$. The formula (5.1) then reduces to the quadratic transformation (5.6) with $j=1$.

If we try to apply the same reasoning to the more general function with $r$ pairs of parameters differing by the positive integers $\left(m_{r}\right)$

$$
{ }_{r+2} F_{r+1}\left(\begin{array}{cc}
a, b, & \left(f_{r}+m_{r}\right) \\
a+b+\frac{1}{2}, & \left(f_{r}\right)
\end{array} \mid X\right),
$$

we obtain from (5.3) a series of $m+1$ terms involving the Gauss functions

$$
F_{k} \equiv{ }_{2} F_{1}\left(\begin{array}{c|c}
a+k, b+k \\
a+b+k+\frac{1}{2}
\end{array} \mid X\right) \quad(0 \leq k \leq m) .
$$

A quadratic transformation for ${ }_{2} F_{1}(\alpha, \beta ; \gamma \mid x)$ exists if and only if any of the quantities

$$
\pm(1-\gamma), \quad \pm(\alpha-\beta), \quad \pm(\alpha+\beta-\gamma)
$$

are such that either one of them equals $\frac{1}{2}$ or two of them are equal $[10$, p. 560]. In this case, the third condition above for the functions $F_{k}$, with $0 \leq k \leq m$, has the form $\alpha+\beta-\gamma=k-\frac{1}{2}$; that is, a quadratic transformation exists only when $k=0$ and $k=1$. Consequently, we are compelled to take $r=1, m=1$ in (5.9). 


\section{References}

[1] A. R. Miller, Certain summation and transformation formulas for generalized hypergeometric series, J. Comput. Appl. Math. 231 (2009) 964-972.

[2] A. R. Miller and R. B. Paris, A generalized Kummer-type transformation for the ${ }_{p} F_{p}(x)$ hypergeometric function, Canadian Math. Bulletin (to appear 2011).

[3] A. R. Miller and R. B. Paris, Euler-type transformations for the generalized hypergeometric function ${ }_{r+2} F_{r+1}(x)$ (submitted for publication 2010).

[4] A. R. Miller and R. B. Paris, Transformation formulas for the generalized hypergeometric function with integral parameter differences (submitted for publication 2010).

[5] A. R. Miller and H. M. Srivastava, Karlsson-Minton summation theorems for the generalized hypergeometric series of unit argument, Integral Transforms and Special Functions (in press 2010).

[6] R. L. Graham, D. E. Knuth and O. Patashnik, Concrete Mathematics, second ed., Addison-Wesley, Upper Saddle River, 1994.

[7] H. Ki and Y.-O. Kim, On the zeros of some generalized hypergeometric functions, J. Math. Anal. Appl. 243 (2000) 249-260.

[8] A. P. Prudnikov, Yu. A. Brychkov and O. I. Marichev, Integrals and Series, vol. 3, Gordon and Breach, New York, 1990.

[9] A. K. Rathie and T. K. Pogány, New summation formula for ${ }_{3} F_{2}\left(\frac{1}{2}\right)$ and a Kummer-type II transformation of ${ }_{2} F_{2}(x)$, Mathematical Communications 13 (2008) 63-66.

[10] M. Abramowitz and I. A. Stegun (Eds.), Handbook of Mathematical Functions, Dover, New York, 1965. 